\title{
CHEBYSHEV-GRÜSS TYPE INEQUALITIES ON TIME SCALES VIA TWO LINEAR ISOTONIC FUNCTIONALS
}

\author{
LUdMILA NiKOlOVA AND SANJA VAROŠANEC
}

Abstract. We give a generalization of the Chebyshev-Grüss inequality by using the concept of derivative on time scales combined with application of the Chebyshev inequality involving two linear isotonic functionals. This approach covers integral case, discrete case, results from fractional and quantum calculus.

Mathematics subject classification (2010): 26E70, 26D15, 26A33.

Keywords and phrases: The Chebyshev-Grüss inequality, fractional integral operator, isotonic linear functional, time scale.

\section{REFERENCES}

[1] R. Agarwal, M. Bohner, A. Peterson, Inequalities on time scales: A survey, Math. Inequal. Appl., 4 (4) (2001), 535-557.

[2] M. ANwAR, R. BIBI, M. Bohner, J. PeČARIĆ, Integral inequalities on time scales via the theory of isotonic linear functionals, Abstr. Appl. Anal. 2001 (2011) Art. ID: 483595, 1-16.

[3] D. Baleanu, S. D. Purohit, Praveen Agarwal, On fractional integral inequalities involving hypergeometric operators, Chin. J. Math. (N. Y.), Volume 2014 (2014), Article ID 609476.

[4] S. Belarbi, Z. Dahmani, On some new fractional integral inequalities, JIPAM. J. Inequal. Pure Appl. Math., 10 (3) (2009), Art. 86.

[5] M. Bohner, A. Peterson, Dynamic Equations on Time Scales: An Introduction with Applications, Birkhäuser, Boston, 2001.

[6] M. Bohner, G. Guseinov, A. Peterson, Introduction to the time scales calculus, Chapter in book: Advances in Dynamic Equations on Time Scales, Birkhäuser, Boston, 2003, pp. 1-15, Eds: M. Bohner and A. Peterson.

[7] K. BRAHIM, S. TAF, On some fractional q-integral inequalities, Malaya Journal of Matematik, 3 (1) (2013), 21-26.

[8] Z. DAHMANI, Some results associated with fractional integrals involving the extended Chebyshev functional, Acta Univ. Apulensis Math. Inform., 27 (2011), 217-224.

[9] S. S. Dragomir, Some integral inequalities of Grüss type, Indian J. Pure Appl. Math., 31 (4) (2000), 397-415.

[10] H. GonsKa, I. RA̧SA AND M. Rusu, Čebyšev-Grüss-type inequalities revisited, Math. Slovaca, 63 (5) (2013), 1007-1024.

[11] G. SH. Guseinov, Integration on time scales, J. Math. Anal. Appl., 285 (1) (2003), 107-127.

[12] D. S. Mitrinović, J. E. PeČArić And A. M. Fink, Classical and new Inequalities in Analysis, Kluwer Academic Publishers, Dordrecht-Boston-London, 1993.

[13] L. Nikolova, S. VAROŠANEC, Chebyshev and Grüss type inequalities involving two linear functionals and applications, Math. Inequal. Appl., 19 (1) (2016), 127-143.

[14] J. PeČARIĆ, On the Ostrowski generalization of Čebyšev's inequality, J. Math. Anal. Appl., 102 (2) (1984), 479-487.

[15] J. PeČArić, S. S. Dragomir And J. SÁndor, On some Grüss type inequalities for isotonic functionals, Rad Hrvat. Akad. Znan. Umj. Mat. Znan., [467] 11 (1994), 41-47.

[16] M. Z. SARIKAYA, N. AKTAN, H. YILDIRIM, On weighted Čebyšev-Grüss type inequalities on time scales, J. Math. Inequal., 2 (2008), 185-195. 
[17] J. TARiboon, S. K. NTOUYAS, Quantum integral inequalities on finite intervals, J. Inequal. Appl., 2014, 2014:121.

[18] S. G. Topal, Rolle's and generalized mean value theorems on time scales, J. Difference Equ. Appl., 8 (4) (2002), 333-344.

[19] W. YANG, Some new Chebyshev and Grüss-type integral inequalities for Saigo fractional integral operators and their q-analogues, Filomat, 29 (6) (2015), 1269-1289.

[20] W. YANG, On weighted q-Čebyšev-Grüss type inequalities, Comput. Math. Appl., 61 (2011), 1342 1347. 